\title{
The Compact Magnetic Field Structures of AGN from Space VLBI Polarization Observations
}

\author{
D. C. Gabuzda
}

Joint Institute for VLBI in Europe, Dwingeloo, The Netherlands

\section{J. L. Gómez}

Instituto de Astrofísica de Andalucía, Granada, Spain

\section{Introduction}

BL Lacertae objects are active galactic nuclei with weak, sometimes undetectable, optical line emission and strong variability in total intensity and linear polarization over a broad range of wavelengths from ultraviolet to radio. It is believed that synchrotron radiation is the dominant emission mechanism virtually throughout the spectrum. Their strong linear polarization makes BL Lac objects prime targets for space VLBI polarization observations.

The parsec-scale radio jets of BL Lacertae objects imaged with VLBI show a number of distinctive features. The most striking is the dominance of transverse magnetic fields on the wide range of scales probed by ground VLBI observations at frequencies from 43 to $5 \mathrm{GHz}$. Some compact VLBI components with transverse fields are undoubtedly relativistic shocks, in which the transverse field has been enhanced by compression (Laing 1980; Hughes, Aller, \& Aller 1989). However, there is growing evidence that we are also detecting the toroidal component of an underlying helical magnetic field associated with the VLBI jet (Gabuzda 1999, Pushkarev \& Gabuzda 2000, Gabuzda \& Pushkarev 2001).

\section{Results}

We summarize the results of our $5-\mathrm{GHz}$ VSOP space-VLBI polarization observations for the four BL Lacertae objects 1803+784, OJ287, 0735+178, and 1334-127 below. The VSOP observations clearly demonstrate that the groundbased VLBI "core" is usually dominated by the emission of bright, compact components in the innermost jet, as suggested by Gabuzda et al. (1994).

$1803+784$. The most remarkable feature of our $5-\mathrm{GHz}$ VSOP images of this source (Gabuzda 1999) is that the VLBI jet curves appreciably, and the polarization electric vector $\chi$ remains aligned with the local jet direction as it changes, indicating that the jet $\mathbf{B}$ field is everywhere transverse. Gabuzda (1999) interpreted this as evidence for a toroidal, possibly helical, B field associated with the VLBI jet of $1803+784$.

OJ287. Our VSOP images show that the ground-based 5-GHz core polarization is dominated by inner-jet emission from a region where the predominant B field is longitudinal (Gabuzda \& Gómez 2001). The mechanism for generating ordered longitudinal fields on these scales is not obvious; the longitudinal field 
may have been enhanced by shear, or may be associated with oblique or conical shocks (e.g., Cawthorne \& Cobb 1990). We had 22 GHz VLBA data about two weeks earlier than the VSOP observations. The core is optically thick at $5 \mathrm{GHz}$ but optically thin at $22 \mathrm{GHz}$, leading to a nearly $90^{\circ}$ flip in its polarization position angle between the two frequencies.

0735+178. We had $15 \mathrm{GHz}$ VLBA data about a month later than our 5$\mathrm{GHz}$ VSOP experiment (Gabuzda, Gómez \& Agudo, in prep.). A spectral-index map demonstrated that the northern part of the jet, which extends roughly toward the northeast, has a steep spectrum, while the southern part of the jet has an inverted spectrum. This suggests the presence of appreciable absorption in the southern VLBI jet at $5 \mathrm{GHz}$. The absorption is concentrated just where the jet makes a sharp bend, possibly suggesting that this bend is associated with an interaction with the surrounding medium (a cloud?).

1334-127. This object is extremely compact. The VSOP image detected polarization in only one feature, which is a bright knot in the inner jet.

\section{Conclusion}

Space VLBI polarization observations represent a unique way to increase resolution without losing sensitivity to steep-spectrum emission. Combined with quasi-simultaneous ground VLBI observations at higher frequencies, they can provide a powerful tool for studying the inner jet regions in the most compact AGN. For each of the four BL Lac objects for which space VLBI polarization observations are available, the VSOP images have yielded interesting and unexpected results, that clearly demonstrate the potential value of space VLBI observations at centimeter wavelengths. There is every reason to expect that future space VLBI polarization observations will yield a wide range of new information about the nature of the brightest jet features and their evolution, the degree to which their magnetic fields are ordered and the mechanisms leading to this ordering, the role of depolarization, and the distribution of thermal plasma near the jet.

\section{References}

Cawthorne, T.V. \& Cobb, W.K. 1990, ApJ, 350, 536-544

Gabuzda, D.C. 1999, New Astronomy Reviews, 43, 691-694

Gabuzda, D.C. \& Gómez 2001, MNRAS, 320, L49

Gabuzda, D.C., Mullan, C.M., Cawthorne, T.V., Wardle, J.F.C., \& Roberts, D.H. 1994, ApJ, 435, 140-161

Gabuzda, D.C. \& Pushkarev, A.B. 2001, in 'Particles and Fields in Radio Galaxies', eds. K. Blundell \& R. Laing, in press

Hughes, P.A., Aller, H.D., \& Aller, M.F. 1989, ApJ, 341, 68-79

Laing, R. A. 1980, MNRAS, 193, 439-449

Pushkarev, A.B. \& Gabuzda, D.C. 2000, in 'Proceedings of the 5th EVN Symposium', in press 